\title{
SOBRE PROJETO DE OBSERVADORES DESACOPLADOS DE PERTURBAÇÃO PARA SISTEMAS DESCRITORES
}

\author{
Vilemar G. da Silva* \\ vilemar@dee.ufma.br
}

Sophie Tarbouriech

tarbour@laas.fr

\author{
Eugênio B. Castelan ${ }^{\dagger}$ \\ eugenio@das.ufsc.br
}

Germain Garcia ${ }^{\ddagger}$

garcia@laas.fr

\author{
*DE.EE-CCET-UFMA \\ Campus universitário do Bacanga-65085-580, São Luís, MA, Brasil \\ $\dagger$ DAS-CTC-UFSC \\ Departamento de Automação e Sistemas - 88040-900, Florianópolis, SC, Brasil \\ ${ }^{\ddagger}$ LAAS-CNRS \\ 7, Av. du Colonel Roche -31077, Toulouse, France
}

\section{RESUMO}

Condições necessárias e suficientes para a existência de observadores de ordem reduzida desacoplados de perturbação para sistemas descritores lineares contínuos são apresentadas. Essas condições são baseadas na solução de uma equação de Sylvester generalizada, sob uma restrição que descreve o desacoplamento de perturbação do estado estimado e numa condição de posto que garante a existência de matrizes para a reconstrução de todas as variáveis de estado, também com desacoplamento de perturbação. Mostra-se que as condições para a existência do observador desejado estão associadas, fundamentalmente, a certas propriedades estruturais do sistema descritor. A partir destes resultados, propõe-se um algoritmo de projeto baseado em técnica de posicionamento de autoestrutura. Os resultados apresentados destinam-se, fundamentalmente, à aplicação em síntese de leis de controle do tipo realimentação de estados estimados. Um exemplo numérico ilustrativo do

\footnotetext{
Artigo submetido em 03/08/2004

1a. Revisão em 03/08/2005

2a. Revisão em 21/09/2006

3a. Revisão em 21/05/2007

Aceito sob recomendação do Editor Associado Prof. Liu Hsu
}

procedimento de projeto é apresentado.

PALAVRAS-CHAVE: Sistemas descritores, Observadores, Ordem mínima, Equação de Sylvester, Posicionamento de Autoestrutura.

\section{ABSTRACT}

Necessary and sufficient conditions for the existence and design of disturbance decoupled reduced-order observers for continuous linear descriptor systems are presented. The observer existence conditions are based on the solution of a generalized Sylvester's equation, under a constraint that describes the disturbance decoupling of the estimated state, and on a rank condition that guarantees the existence of matrices for the reconstruction of all state variables, also with disturbance decoupling. Basically, the design conditions of the desired observer are associated to some structural properties of the descriptor system. From these results, we propose an observer design algorithm that is based on eigenstructure assignment technique. The presented results are useful when an estimated state feedback control law is considered. An illustrative numerical example is reported.

KEYWORDS: Descriptor Systems, Observers, Minimal 
order, Sylvester's equation, Eigenstructure assignment.

\section{INTRODUÇÃO}

A teoria de estimação de estados exerce um papel importante em diferentes campos técnicos e científicos tais como em controle de sistemas dinâmicos (Chen, 1999) (Dai, 1989) (Liu and Patton, 1998), em detecção/diagnóstico de falhas (Liu and Patton, 1998) (Bolivar, 2001) e em filtragem (Nagpal and Khargonekar, 1991; Yaesh and Shaked, 1992). Nas aplicações em controle de sistemas dinâmicos, os observadores (ou estimadores) de estado viabilizam a implementação de leis de controle de realimentação de estados, parcial ou completamente estimados, preservando, em particular, a estabilidade do sistema em malha fechada para ambos os casos. Nesse campo de aplicação, um observador de estados constitui-se em um subsistema dinâmico, excitado pelas entradas e saídas do sistema a controlar. Os estados do observador devem permitir recuperar, assintoticamente, as trajetórias dos estados do sistema ou de uma combinação deles.

Considerando que o sistema a controlar está sujeito a perturbações externas, é desejável que o comportamento dinâmico do observador esteja, parcialmente ou completamente, desacoplado das perturbações, ou satisfaça algum critério de desempenho face à influência dessas perturbações no erro de observação do sistema. Portanto, um observador desacoplado de perturbações externas consiste de um sistema observador que rejeita completamente alguma perturbação considerada do erro de observação.

Embora o desacoplamento (ou rejeição completa) de perturbação possa parecer um requisito muito forte, observadores desacoplados de perturbação podem ser projetados se o sistema considerado verifica algumas condições estruturais, que estão geralmente relacionadas a alguma propriedade de detectabilidade e ao número de saídas medidas comparado ao número de entradas de perturbação. Além disso, algumas propriedades inerentes a um projeto por realimentação de estados, relativas à relação perturbaçãosaída, continuam válidas em malha fechada quando um observador desacoplado de perturbação é utilizado para implementar a lei de controle.

O desacoplamento de perturbações em projeto de observadores de estado foi tratado na literatura científica, principalmente para o caso de sistemas lineares normais (descritos por equações diferenciais ordinárias de primeira ordem), como por exemplo, em (Johnson, 1975) (Bhattacharyya, 1978) (Hou and Muller, 1992a) (Syrmos, 1993) (Liu and Patton, 1998). O desacoplamento de perturbações em observadores para o caso de sistemas descritores (descritos por equações diferenciais de primeira ordem e equações algébricas) foi tratado, por exemplo, em (Yang and Tan, 1989) (Paraskevopoulos et al., 1992) (Hou and Muller, 1992b) (Darouach et al., 1996) (Chu and Mehrmann, 1999a).

O objetivo deste trabalho é abordar o problema de projeto de observadores desacoplados de perturbações externas para sistemas descritores. Relativamente a trabalhos similares, a originalidade da técnica de projeto proposta reside: i) na resolução explícita de uma equação de Sylvester generalizada sujeita a duas condições complementares, e ii) na utilização de sinais auxiliares, definidos em função das entradas de controle e das saídas medidas, respectivamente. $\mathrm{Na}$ definição desses sinais auxiliares, são empregadas matrizes que permitem anular a ação da perturbação sobre as variáveis algébricas do sistema descritor e sobre as variáveis de saída. Já a possibilidade de solução da equação de Sylvester conjuntamente com as condições complementares, é interpretada em termos de propriedades estruturais que devem ser verificadas pelo sistema descritor para que se possa projetar o observador desacoplado de perturbação (ODP).

Este trabalho está organizado da maneira seguinte. Na seção 2 é feita uma apresentação do problema, onde são mostradas as equações do sistema descritor, do observador e dos sinais auxiliares a serem utilizados. Na seção 3 são apresentadas condições necessárias e suficientes para a existência do observador desejado, e uma breve análise considerando a inclusão de uma dada lei de controle de realimentação de estados estimados. Na seção 4: i) são apresentadas e interpretadas as condições estruturais do sistema descritor que permitem o projeto do observador, ii) relacionam-se os quatro casos possíveis relativos à ação das perturbações sobre as variáveis do sistema, indicando-se a estrutura/ordem mínima do observador mais adequada para cada caso. Na seção 5 propõe-se um algoritmo de cálculo com base nos resultados anteriores e mostram-se os resultados numéricos e de simulação, para um exemplo de sistema descritor. Em seguida apresentam-se as conclusões.

\section{APRESENTAÇÃO DO PROBLEMA}

Seja um sistema descritor linear contínuo sujeito a perturbações externas descrito por:

$$
\begin{aligned}
E \dot{x}(t) & =A x(t)+B u(t)+B_{w} w(t) \\
y(t) & =C x(t)+D_{w} w(t)
\end{aligned}
$$

onde $x \in \Re^{n}, y \in \Re^{p}, u \in \Re^{m}$ e $w \in \Re^{r}$ são, respectivamente, os vetores de estados, de saídas medidas, de entradas de controle e de entradas de perturbação. A matriz $E \in \Re^{n \times n}$ é tal que posto $(E)=q<n$, que caracteriza um modelo de sistema descritor linear, $A \in \Re^{n \times n}, B \in \Re^{n \times m}$, $\operatorname{com} \operatorname{posto}(B)=m, C \in \Re^{p \times n}, \operatorname{com} \operatorname{posto}(C)=p$, 
$B_{w} \in \Re^{n \times r}, \operatorname{com} \operatorname{posto}\left(B_{w}\right)=r$ e $D_{w} \in \Re^{p \times r}$, com $\operatorname{posto}\left(D_{w}\right)=\tilde{r}<p^{1}$.

Seja $L \in \Re^{(n-q) \times n}$ uma matriz de posto completo que verifica

$$
L E=0
$$

Defina $B_{d} \in \Re^{(n-q) \times r}$ e o escalar $\bar{r}$ por

$$
B_{d}=L B_{w} \text { e } \bar{r}=\operatorname{posto}\left(B_{d}\right)<n-q
$$

Para efeito de implementação de leis de controle do tipo realimentação de estados estimados $u(t)=F \hat{x}(t)+$ $v(t)$, onde $v(t)$ representa uma nova entrada de controle ou de referência, considera-se um observador de estados representado sob a forma:

$$
\begin{aligned}
& \dot{z}(t)=H z(t)+T B u(t)-Z y(t) \\
& \hat{x}(t)=S z(t)+\bar{N} \bar{y}(t)+\tilde{N} \tilde{y}(t)
\end{aligned}
$$

onde $z \in \Re^{k}$ e $\hat{x} \in \Re^{n}$ são os vetores de estados estimados e de estados observados, respectivamente.

Os vetores $\bar{y} \in \Re^{n-q-\bar{r}}$ e $\tilde{y} \in \Re^{p-\tilde{r}}$ representam sinais auxiliares gerados a partir das entradas de controle e das saídas medidas, como segue:

$$
\bar{y}=-\bar{Q} L B u(t)
$$

$\operatorname{com} \bar{Q} \in \Re^{(n-q-\bar{r}) \times(n-q)}$ tal que $\bar{Q} B_{d}=0, \operatorname{posto}(\bar{Q})=$ $n-q-\bar{r}, \mathrm{e}$

$$
\tilde{y}=\tilde{Q} y(t)
$$

$\operatorname{com} \tilde{Q} \in \Re^{(p-\tilde{r}) \times p}$ tal que $\tilde{Q} D_{w}=0$ e $\operatorname{posto}(\tilde{Q})=p-\tilde{r}$.

A figura 1 ilustra o sistema em malha fechada, contendo o sistema original, o observador e um controlador por realimentação de estados estimados. Observe que a parte dinâmica do observador, descrita por sua equação de estados (5), é similar à dos observadores de ordem reduzida para sistemas normais (ver por exemplo (Chen, 1999)). Como pode ser visto nas duas seções seguintes, a definição do sinal $\bar{y}$ permite levar em conta a parte algébrica das variáveis de estado do sistema descritor, no projeto do observador.

O problema tratado na seqüência destina-se ao projeto de observadores (5)-(6) para sistemas descritores (1)-(2), sem influência das perturbações $w(t)$. Deseja-se verificar sob quais condições é possível determinar as matrizes $H, T$,

\footnotetext{
${ }^{1} \mathrm{O}$ conjunto de pólos (finitos e infinitos) de um sistema descritor corresponde ao conjunto de autovalores generalizados do par $(E, A)$. Se o sistema é regular, o conjunto dos pólos finitos, denotado $\sigma(E, A)$, é formado pelas raízes da equação característica $\operatorname{det}(\lambda E-A)=0$ (Castelan, 2005; Dai, 1989).
}

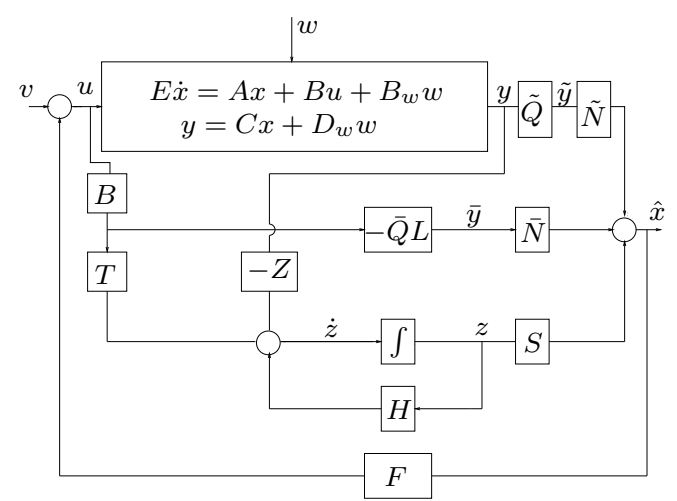

Figura 1: Sistema em malha fechada com realimentação de estados estimados.

$Z, S, \bar{N}$ e $\tilde{N}$ tais que o erro entre $x(t)$ e $\hat{x}(t)$ é nulo em regime estacionário, para qualquer $w(t) \in \Re^{r}$. Um sistema observador de estados sob essas condições será dito um Observador Desacoplado de Perturbações (ODP).

A ordem do observador (5)-(6) é determinada não somente em função da ordem dinâmica do sistema descritor, $q$, e do número de saídas, $p$, mas também de como a perturbação atua sobre a parte algébrica das variáveis de estado (Dai, 1989) e sobre as variáveis de saída, através das matrizes $\bar{Q}$ e $\tilde{Q}$, respectivamente. Em particular, se $L B_{w}=B_{d}=0$ e $D_{w}=0$, tem-se $\bar{Q}=I_{n-q}$ e $\tilde{Q}=I_{p}$, e a ordem mínima é $k=q-p$ (Castelan et al., 2004). Nas outras situações, a estrutura proposta também permite atingir a menor ordem possível.

\section{OBSERVADORES DESACOPLADOS DE PERTURBAÇÕES}

Nesta seção são apresentadas as condições para a existência de um ODP de ordem reduzida $k \geq q-p$, essencialmente descritas pela possibilidade de solução de uma equação de Sylvester conjuntamente com uma condição para o desacoplamento das perturbações. Ao final, considera-se a uma lei de controle de realimentação de estados estimados, e faz-se uma breve análise para o sistema de malha fechada correspondente.

Proposição 3.1 Dado um sistema descritor (1)-(2), existe um observador de ordem reduzida $k$ na forma (5)-(6) tal que, para qualquer perturbação $w \in \Re^{r}$ :

i) o erro de estimação $\varepsilon(t)=z(t)-T E x(t)$ verifica: $\varepsilon(t)=e^{H t} \varepsilon(0), \forall t \geq 0$ e $\lim _{t \rightarrow \infty} \varepsilon(t)=0, \forall z(0)$,
$E x(0) ; \mathrm{e}$

ii) para matrizes $S, \bar{N}$ e $\tilde{N}$, adequadamente calculadas, o 
estado estimado $\hat{x} \in \Re^{n}$ verifica $\lim _{t \rightarrow \infty}[x(t)-\hat{x}(t)]=0$;

se e somente se é possível resolver a equação de Sylvester generalizada

$$
T A-H T E=-Z C, \operatorname{com} \sigma(H) \subset \mathcal{C}^{-}
$$

sob as condições adicionais:

$$
\begin{gathered}
T B_{w}+Z D_{w}=0 \\
\text { posto }\left(\left[\begin{array}{c}
T E \\
\bar{Q} L A \\
\tilde{Q} C
\end{array}\right]\right)=n
\end{gathered}
$$

onde: $H \in \Re^{k \times k}, T \in \Re^{k \times n}$ e $Z \in \Re^{k \times p}$; e $\sigma(H)$ denota o espectro de $H$.

\section{Demonstração:}

Considere o erro de estimação

$$
\varepsilon(t)=z(t)-T E x(t)
$$

Sua derivada, em relação ao tempo, é dada por:

$$
\dot{\varepsilon}(t)=\dot{z}(t)-T E \dot{x}(t)
$$

Fazendo substituições entre as equações (1), (2), (5) e (13) obtém-se, após breve simplificação:

$$
\dot{\varepsilon}(t)=H z(t)-(Z C+T A) x(t)-\left(T B_{w}+Z D_{w}\right) w(t)
$$

Observa-se que a parte (i) da proposição é satisfeita se e somente se a dinâmica do erro de estimação for descrita pelo sistema dinâmico assintoticamente estável:

$$
\dot{\varepsilon}(t)=H \varepsilon(t), \operatorname{com} \sigma(H) \subset \mathcal{C}^{-}
$$

Portanto, comparando-se (14) com (15), conclui-se que as condições (9) e (10) são necessárias e suficientes para o desacoplamento de perturbação do erro de estimação relativo à parte $(i)$ da proposição.

Para a demonstração da parte (ii), supor que (11) também é verificada e considerar (6) escrita na forma

$$
\hat{x}=\left[\begin{array}{lll}
S & \bar{N} & \tilde{N}
\end{array}\right]\left[\begin{array}{c}
z(t) \\
\bar{y}(t) \\
\tilde{y}(t)
\end{array}\right]
$$

Multiplicando, à esquerda, a equação (1) por $\bar{Q} L$ e lembrando que, por definição, $L E=0$, obtém-se

$$
0=\bar{Q} L A x(t)+\bar{Q} L B u(t)+\bar{Q} L B_{w} w(t)
$$

Também por definição, $\bar{Q} L B_{w} w(t)=0$. Então, combinando (7) com (17) resulta em

$$
\bar{y}=\bar{Q} L A x(t)
$$

De (2) e (8), lembrando que $\tilde{Q} D_{w}=0$, obtém-se

$$
\tilde{y}=\tilde{Q} C x(t)
$$

Conforme a demonstração da parte $(i), \lim _{t \rightarrow \infty} \varepsilon(t)=0$. Então, no limite quando $t \rightarrow \infty$, as equações (12), (18) e (19) podem ser escritas conjuntamente como

$$
\lim _{t \rightarrow \infty}\left[\begin{array}{c}
z(t) \\
\bar{y}(t) \\
\tilde{y}(t)
\end{array}\right]=\left[\begin{array}{c}
T E \\
\bar{Q} L A \\
\tilde{Q} C
\end{array}\right] \lim _{t \rightarrow \infty} x(t)
$$

Tomando o limite de (16), com $t \rightarrow \infty$, e combinando o resultado com (20), obtém-se:

$$
\begin{aligned}
& \lim _{t \rightarrow \infty}[x(t)-\hat{x}(t)]= \\
&=\left(I_{n}-\left[\begin{array}{ll}
S \bar{N} & \tilde{N}
\end{array}\right]\left[\begin{array}{c}
T E \\
\bar{Q} L A \\
\tilde{Q} C
\end{array}\right]\right) \lim _{t \rightarrow \infty} x(t) \\
& \quad=\left(I_{n}-M^{\dagger} M\right) \lim _{t \rightarrow \infty} x(t)
\end{aligned}
$$

em que $M^{\dagger}=\left[\begin{array}{ccc}S & \bar{N} & \tilde{N}\end{array}\right] \in \Re^{n \times(k+n+p-q-\bar{r}-\tilde{r})}$ é, em geral, uma pseudo-inversa à esquerda de $M=$ $\left[\begin{array}{lll}(T E)^{\prime} & (\bar{Q} L A)^{\prime} & (\tilde{Q} C)^{\prime}\end{array}\right]^{\prime}$.

Logo, independentemente do valor de $\lim _{t \rightarrow \infty} x(t)$, o erro de observação $x(t)-\hat{x}(t)$ é nulo em regime permanente, se e somente se for possível anular o termo entre parênteses na relação anterior ou, equivalentemente, se for possível calcular as matrizes $S, \bar{N}$ e $\tilde{N}$ de modo que

$$
M^{\dagger} M=I_{n}
$$

Isto pode ser realizado, se e somente se a condição (11) é verificada.

A resolução de (9)-(10) está associada, fundamentalmente, ao número de entradas de perturbação e a propriedades estruturais ligadas ao sistema $\left(E, A, C, B_{w}, D_{w}\right)$, a serem discutidas posteriormente.

Da demonstração acima, é importante salientar o papel dos sinais auxiliares $\bar{y}$ e $\tilde{y}$, definidos a partir das matrizes $B_{d}=L B_{w}$ e $D_{w}$, fundamentais para a obtenção do erro de estados estimados desacoplado da perturbação. De fato, se tivéssemos $\bar{Q} B_{d} \neq 0$ e $\tilde{Q} D_{w} \neq 0$, então, ao invés de (20), 
teríamos

$$
\begin{aligned}
& \lim _{t \rightarrow \infty}\left[\begin{array}{l}
z(t) \\
\bar{y}(t) \\
\tilde{y}(t)
\end{array}\right]= \\
= & \lim _{t \rightarrow \infty}\left(\left[\begin{array}{c}
T E \\
\bar{Q} L A \\
\tilde{Q} C
\end{array}\right] x(t)\right)+\lim _{t \rightarrow \infty}\left(\left[\begin{array}{c}
0 \\
\bar{Q} L B_{w} \\
\tilde{Q} D_{w}
\end{array}\right] w(t)\right)
\end{aligned}
$$

o que implicaria, em particular, $\lim _{t \rightarrow \infty}[x(t)-\hat{x}(t)] \neq 0$ se $\lim _{t \rightarrow \infty} w(t) \neq 0$.

Em função da definição dos sinais auxiliares $\bar{y}$ e $\tilde{y}$, descrevem-se na seção seguinte diferentes casos para os quais pode-se considerar o projeto de observadores desacoplados de perturbação sob a estrutura proposta, associando-se, em particular a definição da ordem do observador à condição (11). Note que, em função de (11), a ordem do observador deve verificar $k \geq q-p+\bar{r}+\tilde{r}$ (veja também (21) e (22)). Então, a ordem mínima possível para o observador ocorre no caso em que a matriz $M=$ $\left[\begin{array}{lll}(T E)^{\prime} & (\bar{Q} L A)^{\prime}(\tilde{Q} C)^{\prime}\end{array}\right]^{\prime}$, de posto $n$, é quadrada e portanto inversível, de modo que $M^{\dagger}=M^{-1}$.

Para finalizar esta seção, considera-se a utilização do observador desacoplado de perturbação para a implementação de uma lei de controle do tipo realimentação de estados estimados, dada por

$$
u(t)=F \hat{x}(t)+v(t)
$$

em que $F \in \Re^{m \times n}$ é uma matriz de ganho de realimentação e $v(t)$ é uma nova entrada de controle ou de referência. Supõe-se que a matriz $F$ é tal que o par $(E, A+B F)$ é fortemente estável, ou seja: assintoticamente estável, regular e livre de modos impulsivos (Varga, 1995).

O sistema composto em malha-fechada pode ser representado (ver Apêndice) por:

$$
\begin{gathered}
{\left[\begin{array}{cc}
E & 0 \\
0 & I_{k}
\end{array}\right]\left[\begin{array}{c}
\dot{x}(t) \\
\dot{\varepsilon}(t)
\end{array}\right]=} \\
=\left[\begin{array}{cc}
A+B F & B F S \\
0 & H
\end{array}\right]\left[\begin{array}{l}
x(t) \\
\varepsilon(t)
\end{array}\right]+ \\
+\left[\begin{array}{l}
B \\
0
\end{array}\right] v(t)+\left[\begin{array}{c}
B_{w} \\
0
\end{array}\right] w(t) \\
y(t)=\left[\begin{array}{ll}
C & 0
\end{array}\right]\left[\begin{array}{l}
x(t) \\
\varepsilon(t)
\end{array}\right]+D_{w} w(t)
\end{gathered}
$$

O sistema (25)-(26) é também um sistema descritor, no qual, em particular, posto $\left(\left[\begin{array}{cc}E & 0 \\ 0 & I_{k}\end{array}\right]\right)=q+k$. A partir das estruturas das matrizes em (25)-(26), verifica-se que:

1) pela propriedade da separação, o sistema em malha fechada possui $q+k$ pólos finitos assintoticamente estáveis, dados por $\sigma(E, A+B F) \dot{\cup} \sigma(H) \subset \mathcal{C}^{-}$, e portanto é fortemente estável ;

2) devido às características do observador desacoplado de perturbação, a ação da perturbação sobre o sistema em malha fechada ocorre diretamente através de $B_{w}$ e $D_{w}$ e, como no caso de uma realimentação de estados medidos, $u(t)=F x(t)+v(t)$, o comportamento perturbaçãosaída, considerando-se $x(0)$ tal que $E x(0)=0$, pode ser representado pela matriz de transferência:

$$
G_{w y}=C(s E-A-B F)^{-1} B_{w}+D_{w}
$$

3) em regime transitório, o comportamento dinâmico do sistema em malha fechada é influenciado pela dinâmica atribuída ao observador, através das matrizes $H$ e $S$ obtidas pelo projeto do observador, e pelas entradas $w(t)$ e $v(t)$.

\section{RESULTADOS COMPLEMENTARES}

A seguir apresentam-se as hipóteses que permitem o cálculo de um ODP. Justificativas para a utilização dessas hipóteses são apresentadas nas subseções seguintes.

Hipótese 4.1 O sistema (1)-(2):

i) possui menos entradas de perturbação que saídas medidas, ou seja:

$$
r<p
$$

ii) é fortemente zero-detectável, o que requer as duas condições seguintes:

$$
\begin{aligned}
& \operatorname{posto}\left(\left[\begin{array}{cc}
A-\lambda E & B_{w} \\
C & D_{w}
\end{array}\right]\right)=n+r, \\
& \forall \lambda \notin \mathcal{C}, \lambda \text { finito }
\end{aligned}
$$

$$
\text { posto }\left(\left[\begin{array}{c}
E \\
L A \\
C
\end{array}\right]\right)=n
$$

iii) e, adicionalmente:

$$
\text { posto }\left(\left[\begin{array}{c}
L A \\
C
\end{array}\right]\right)=n-q+p
$$

Em geral, para a construção de observadores para sistemas descritores, é necessário que o par $(E, A)$ seja fortemente detectável, o que requer a satisfação da condição 
posto $\left(\left[\begin{array}{c}A-\lambda E \\ C\end{array}\right]\right)=n, \forall \lambda \notin \mathcal{C}, \lambda$ finito, em conjunto com (30), (veja, por exemplo, (Castelan and Silva, 2005)). Então, a condição (29), sob a condição estrutural (28), constitui-se numa adaptação que permite resolver a equação de Sylvester (9) sob a condição de desacoplamento (10). Note que a condição (30) também é necessária para que (11) possa ser verificada via a síntese da matriz $T$ do observador. Por último, a condição adicional (31) está associada, fundamentalmente, à determinação da ordem do observador.

\subsection{Condições estruturais para solução}

Técnica padrão de posicionamento de autoestrutura pode ser usada para resolver a equação de Sylvester (9) sob a condição de desacoplamento (10) (Liu and Patton, 1998). Por exemplo, se considerarmos $\sigma(H)=\left\{\pi_{1}, \pi_{2}, \ldots, \pi_{k}\right\} \subset$ $\mathcal{C}^{-}, \operatorname{com} \pi_{i+1}=\pi_{i}^{*}$ se $\operatorname{Imag}\left(\pi_{i}\right) \neq 0^{2}$, as matrizes $T$ e $Z$ podem ser formadas a partir dos vetores $\tau_{i} \in \mathcal{C}^{n}$ e $\zeta_{i} \in \mathcal{C}^{p}$ que verificam:

$$
\left[\begin{array}{cc}
\tau_{i}^{\prime} & \zeta_{i}^{\prime}
\end{array}\right]\left[\begin{array}{cc}
A-\pi_{i} E & B_{w} \\
C & D_{w}
\end{array}\right]=\left[\begin{array}{ll}
0 & 0
\end{array}\right]
$$

na qual $\tau_{i+1}=\tau_{i}^{*}$ e $\zeta_{i+1}=\zeta_{i}^{*}$ se $\operatorname{Imag}\left(\pi_{i}\right) \neq 0$.

A existência de vetores não-nulos que permitem a escolha arbitrária dos autovalores $\pi_{i}$ está relacionada às propriedades estruturais do sistema representado por $\left(E, A, C, B_{w}, D_{w}\right) \mathrm{e}$ que podem ser descritas a partir da matriz do sistema $P(\lambda)$, de dimensão $(n+p) \times(n+r)$, dada por:

$$
P(\lambda)=\left[\begin{array}{cc}
A-\lambda E & B_{w} \\
C & D_{w}
\end{array}\right]
$$

Para todo valor arbitrário de $\pi_{i} \in \mathcal{C}$, a existência de um vetor não-nulo $\left[\begin{array}{c}\tau_{i} \\ \zeta_{i}\end{array}\right]$ que satisfaz (32) é garantida se o número de entradas de perturbações é menor que o número de saídas medidas, ou seja, se a condição (i) da Hipótese 4.1 é satisfeita. Observe, entretanto, que se para algum valor particular de $\lambda$ tivermos posto $(P(\lambda))<n+r$, este valor deverá fazer parte obrigatoriamente do espectro da matriz $H$. Quando $r \geq p$, a matriz $P(\lambda)$ perde posto somente para valores particulares de $\lambda$ e a resolução de (9)-(10) não é geralmente possível.

A condição (30) corresponde à detectabilidade dos pólos infinitos do sistema representado por $(E, A, C)$ (Castelan and Silva, 2005). Além disso, utilizando-se a desigualdade de Sylvester (Chen (1999), pag.207) sobre o posto de produto de matrizes, pode-se verificar que a condição (30) é necessária para que a condição (11) possa ser satisfeita.

\footnotetext{
${ }^{2} \operatorname{Imag}\left(\pi_{i}\right)$ denota a parte imaginária de $\pi_{i}$
}

\subsection{Ordem do Observador}

Na classificação apresentada a seguir, consideram-se certas propriedades das matrizes $B_{d}=L B_{w}$ e $D_{w}$ e, a partir delas, identificam-se a ordem mínima do ODP em quatro casos possíveis.

Observe, inicialmente, que a ordem mínima do ODP é determinada pelo número mínimo de linhas linearmente independentes que deverá ter o produto $T E$, para que a condição de posto (11) seja satisfeita. Então, ela é determinada pela relação seguinte:

$$
k_{\text {min }}=\operatorname{posto}(T E)=n-\operatorname{posto}\left(\left[\begin{array}{c}
\bar{Q} L A \\
\tilde{Q} C
\end{array}\right]\right)
$$

Por hipótese, a condição (31) é verificada. Então, utilizandose a desigualdade de Sylvester (Chen (1999), pag.207) sobre o posto de produto de matrizes, tem-se que

$$
\text { posto }\left(\left[\begin{array}{c}
\bar{Q} L A \\
\tilde{Q} C
\end{array}\right]\right)=n-q-\bar{r}+p-\tilde{r}
$$

Assim, de (34) e (35), resulta:

$$
k_{\text {min }}=q-p+\bar{r}+\tilde{r}
$$

Levando em conta a estrutura das matrizes $B_{d}=L B_{w}$ e $D_{w}$, quatro casos distintos podem ser identificados. Então, utilizando (36), a ordem mínima do ODP associado à cada caso é determinada como segue:

Caso 1 Se $B_{d} \neq 0$ e $D_{w} \neq 0$ utiliza-se um observador de ordem mínima $k=q-p+\bar{r}+\tilde{r}$.

Caso 2 Se $B_{d}=0$ e $D_{w}=0$ utiliza-se um observador de ordem mínima $k=q-p$, no qual $\bar{Q} \in \Re^{(n-q) \times(n-q)}$ e $\tilde{Q} \in \Re^{p \times p}$ são matrizes de posto completo. Por simplicidade, pode-se escolher $\bar{Q}=I_{n-q}$ e $\tilde{Q}=I_{p}$.

Caso 3 Se $B_{d}=0$ e $D_{w} \neq 0$ utiliza-se um observador de ordem $k=q-p+\tilde{r}$, no qual $\bar{Q}=I_{n-q}$.

Caso 4 Se $B_{d} \neq 0$ e $D_{w}=0$ utiliza-se um observador de ordem reduzida $k=q-p+\bar{r}$, para o qual $\tilde{Q}=I_{p}$.

\subsection{Discussão complementar}

Como comentado anteriormente, na Hipótese 4.1, a condição (29) se reduz à condição de detectabilidade dos pólos finitos do sistema descritor se o desacoplamento de perturbações não é considerado (Castelan and Silva, 2005). Além disso, a verificação da condição (30), que corresponde à detectabilidade dos pólos infinitos do sistema representado 
por $(E, A, C)$, é fundamental para que os valores calculados para as matrizes $T, \bar{Q}$ e $\tilde{Q}$ satisfaçam a condição (11).

Em relação à condição adicional (31), a partir da qual definese a ordem do observador, cabe ressaltar que ela pode ser relaxada para posto $\left(\left[\begin{array}{c}L A \\ C\end{array}\right]\right)=n-q+p-d$. Neste caso, a deficiência de posto representada por $d$ deve ser compensada pelo acréscimo correspondente na ordem mínima do observador, em cada um dos casos descritos anteriormente.

Quanto à estrutura utilizada na definição do observador, destaca-se como original a utilização dos sinais auxiliares definidos em função das entradas de controle e das saídas medidas, conforme equações (7) e (8) respectivamente, o que se constitui na idéia chave para anular os termos decorrentes das perturbações no erro de observação. Com isto, a ordem do observador é determinada, integradamente entre os casos 1 a 4 da Seção 4.2, em função das matrizes que descrevem a dinâmica das perturbações, o que é, de fato, um resultado singular.

A aplicação da equação de Sylvester generalizada (9), do observador com dinâmica descrita sob a forma (5) e a ordem reduzida do observador são características centrais deste trabalho encontradas também em trabalhos similares: i) não considerando o desacoplamento de perturbações (Verhaegen and dooren, 1986; Muller and Hou, 1993; Darouach and Boutayeb, 1995; Castelan and Silva, 2005), e ii) considerando o desacoplamento de perturbações (Yang and Tan, 1989; Darouach et al., 1996; Chu and Mehrmann, 1999b; Koenig and Mammar, 2002; Castelan et al., 2004).

É importante salientar que, sob o ponto de vista das hipóteses que indicam como as perturbações externas podem agir sobre os atuadores e sensores de um sistema descritor, os resultados propostos no presente trabalho são mais abrangentes que seus similares citados no item (ii) anterior. Esta abrangência refere-se ao fato de que no presente trabalho são previstos quatro casos para o par de matrizes de perturbação $B_{w}, D_{w}$, relacionados ao final da seção anterior.

\section{ALGORITMO E EXEMPLO}

Com base nos resultados anteriores é proposto na seqüência um algoritmo para projeto de ODP de ordem mínima para sistemas descritores que satisfazem a Hipótese 4.1. Um exemplo numérico é apresentado na Subseção 5.2.

\subsection{Algoritmo}

Passo 1 Determinar a ordem mínima do observador, $k_{\text {min }}$, utilizando a equação (36) dentre os casos possíveis $1 a$
4. Definir o espectro de autovalores desejados para o observador: $\sigma(H)=\Pi=\left\{\pi_{1}, \pi_{2}, \ldots, \pi_{k_{m i n}}\right\}$.

Passo 2 Para cada $\pi_{i}$, encontrar $\tau_{i} \in \mathcal{C}^{n}$ e $\zeta_{i} \in \mathcal{C}^{p}$ que verificam (32). As linhas da matriz $T \in \Re^{k \times n}$, denotadas por $T_{i}$, são formadas a partir dos vetores $\tau_{i}$ como segue: se $\pi_{i} \in \Re$, então $T_{i}=\tau_{i}^{\prime}$; se $\pi_{i} \in \mathcal{C}$, considera-se $\pi_{i+1}=\pi_{i}^{*}$ e $T_{i}=\mathbb{R e}\left(\tau_{i}^{\prime}\right), T_{i+1}=$ $\operatorname{Imag}\left(\tau_{i}^{\prime}\right)$. De maneira similar, encontra-se a matriz $Z$. A matriz $H$ correspondente, na forma de Jordan, é $H=\operatorname{diag}\left\{D_{i}\right\}$ onde $D_{i}=\pi_{i}$ se $\pi_{i} \in \Re$, e $D_{i}=\left[\begin{array}{cc}\mathbb{R} e\left\{\pi_{i}\right\} & \mathbb{I m a g}\left\{\pi_{i}\right\} \\ -\mathbb{I m a g}\left\{\pi_{i}\right\} & \mathbb{R} e\left\{\pi_{i}\right\}\end{array}\right]$ se $\pi_{i} \in \mathcal{C}$.

Passo 3 Encontrar as matrizes $\bar{Q}$ e $\tilde{Q}$ que verificam $\bar{Q} B_{d}=$ 0 e $\tilde{Q} D_{w}=0$, respectivamente.

Passo 4 Calcular as matrizes $S, \bar{N}$ e $\tilde{N}$ utilizando a equação (22), $\operatorname{com} M^{\dagger}=M^{-1}$.

É importante lembrar que os vetores $\tau_{i}$, determinados no Passo 2, devem formar um conjunto de vetores linearmente independentes, além de garantir $\operatorname{rank}(T E)=k_{\text {min }}$. Este tipo de requisito, comumente encontrado em técnicas de projeto via posicionamento de autoestrutura, pode ser em geral atendido escolhendo-se valores distintos para os elementos do conjunto $\Pi$ (veja, por exemplo, (Castelan, 2005)). Além disso, pode-se fazer uma verificação numérica do atendimento deste requisito ao longo dos cálculos realizados no Passo 2. Por construção, se posto(TE) $=$ $k_{m i n}$, então a condição (11) é verificada e $M$, em (22), é uma matriz quadrada e inversível.

Deve-se destacar o fato de que a partir da definição de um ODP, é possível determinar novos parâmetros para o observador com o objetivo, por exemplo, de melhorar o condicionamento da matriz não-singular envolvida na condição (11), como proposto em (Silva, 2005), a partir dos graus de liberdade existentes na escolha das matrizes $\bar{Q}$ e $\tilde{Q}$. Além disso, no Caso (ii), em que $B_{d}=0$ e $D_{w}=0$, a síntese do ODP pode ser realizada via uma técnica de posicionamento regional de pólos em regiões do tipo LMI (Castelan and Silva, 2005; Silva, 2005). 


\subsection{Exemplo}

Considerar o sistema descritor sob perturbações, que verifica a Hipótese 4.1, representado pelas matrizes:

$$
\begin{gathered}
E=\left[\begin{array}{rrrrrr}
1 & 0 & 0 & 0 & 0 & 0 \\
0 & 1 & 0 & 0 & 0 & 0 \\
0 & 0 & 1 & 0 & 0 & 0 \\
0 & 0 & 0 & 1 & 0 & 0 \\
0 & 0 & 0 & 0 & 0 & 0 \\
0 & 0 & 0 & 0 & 0 & 0
\end{array}\right], \\
A=\left[\begin{array}{rrrrrr}
0 & 1 & 0 & 0 & 1 & 1 \\
1 & 1 & 0 & 1 & -1 & 0 \\
0 & 0 & 1 & 1 & 0 & 1 \\
0 & 0 & 1 & -1 & 0 & 0 \\
0 & 0 & 0 & 1 & 1 & 0 \\
0 & 0 & 0 & 0 & 0 & 1
\end{array}\right], \\
C=\left[\begin{array}{cccccc}
1 & 1 & 0 & -1 & 0 & 0 \\
0 & 0 & -1 & 0 & 1 & 1
\end{array}\right], \\
B_{w}^{\prime}=\left[\begin{array}{lllll}
1 & 1 & 1 & 1 & {\left[B_{d}^{\prime}\right.}
\end{array}\right] .
\end{gathered}
$$

Para ilustrar a utilização do algoritmo para os quatro casos enunciados na seção 4.2 , supõe-se que $B_{d}=L B_{w}$ pode assumir os valores $B_{d}^{\prime}=\left[\begin{array}{ll}1 & 1\end{array}\right]$ ou $B_{d}^{\prime}=\left[\begin{array}{ll}0 & 0\end{array}\right]$. $\mathrm{Da}$ mesma forma, $D_{w}^{\prime}=\left[\begin{array}{ll}0.7382 & 0.1763\end{array}\right] \mathrm{ou}$ $D_{w}^{\prime}=\left[\begin{array}{ll}0 & 0\end{array}\right]$. Os autovalores utilizados foram: caso 1) $\{-7 \pm 2 i,-8 \pm 3 i\}$, caso 2) $\{-7 \pm 2 i\}$, casos 3 $e$ 4) $\{-7,-8 \pm 2 i\}$. Para os dados acima, foram obtidas as

\begin{tabular}{|c|c|c|c|c|c|}
\hline Caso & \multicolumn{2}{|c|}{$\bar{Q}$} & \multicolumn{2}{|c|}{$\tilde{Q}$} & $\begin{array}{c}\text { Ordem } \\
k\end{array}$ \\
\hline 1 & {$[-0.7071$} & $0.7071]$ & {$[-0.2323$} & 0.9726 & 4 \\
\hline 2 & {$\left[\begin{array}{l}1 \\
0\end{array}\right.$} & $\left.\begin{array}{l}0 \\
1\end{array}\right]$ & {$\left[\begin{array}{l}1 \\
0\end{array}\right.$} & $\left.\begin{array}{l}0 \\
1\end{array}\right]$ & 2 \\
\hline 3 & {$\left[\begin{array}{l}1 \\
0\end{array}\right.$} & $\left.\begin{array}{l}0 \\
1\end{array}\right]$ & {$[-0.2323$} & 0.9726 & 3 \\
\hline 4 & {$[-0.7071$} & $0.7071]$ & {$\left[\begin{array}{l}1 \\
0\end{array}\right.$} & $\left.\begin{array}{l}0 \\
1\end{array}\right]$ & 3 \\
\hline
\end{tabular}
matrizes do ODP, mostradas nas tabelas 1 e 2. As matrizes $S, \bar{N}$ e $\tilde{N}$ podem ser calculadas diretamente da equação (22).

Tabela 1: Matrizes relativas aos sinais auxiliares: $\bar{y}=\bar{Q} B_{d} u$ e $\tilde{y}=\tilde{Q} y$.

Visando ilustrar a validade do método proposto, considere o Caso 1 , em que $B_{d} \neq 0, D_{w} \neq 0$. Além do ODP correspondente a este caso, relacionado nas Tabelas $1 e$
2, é utilizado, para comparação, um outro observador com estrutura similar ao ODP, mas que atende apenas ao requisito de desacoplamento parcial da perturbação relativamente ao erro de estimação $z(t)-T E x(t)$. Assim, este observador compartilha as mesmas matrizes $H, T$ e $Z$ do ODP, o que implica na satisfação da condição de desacoplamento $T B_{w}+$ $Z D_{w}=0$, mas as matrizes $\bar{Q}$ e $\tilde{Q}$ são tais que $\bar{Q} B_{d} \neq 0$, $\tilde{Q} D_{w} \neq 0$, ou seja, elas não satisfazem as condições para a obtenção do desacoplamento da perturbação relativamente ao erro de observação, $x(t)-\hat{x}(t)$, no caso em consideração. Na obtenção das curvas de simulação temporal, as condições iniciais utilizadas para o sistema descritor e o observador foram $x(0)=\left[\begin{array}{llllll}0 & 1 & 0 & 0 & 0 & 0\end{array}\right]^{\prime} \mathrm{e} z(0)=0$, respectivamente. Na figura 2 mostram-se as curvas dos erros de estimação e de observação, para o ODP e para o observador com desacoplamento parcial. Observa-se que os erros de estimação e observação de um ODP convergem aos correspondentes estados nulos, conforme Figura $2(a)$, enquanto apenas o erro de estimação converge ao estado nulo (o mesmo não ocorrendo com o erro de observação) para o observador com desacoplamento parcial, conforme Figura 2 (b).

\section{CONCLUSÕES}

Foram apresentadas condições para a existência de observadores de ordem reduzida para sistemas descritores, desacoplados de pertubações externas. Tais condições se basearam na solução de uma equação de Sylvester generalizada sob duas restrições adicionais. A primeira é uma restrição matricial que descreve o desacoplamento de perturbações do erro de estimação, podendo ser resolvida conjuntamente com a equação de Sylvester. A segunda é uma restrição de posto necessária para a reconstrução das variáveis de estado, com desacoplamento de perturbações do erro de observação. Foram definidos sinais auxiliares em função das entradas de controle e das saídas medidas, respectivamente, sendo utilizadas matrizes ortogonais às matrizes que descrevem a dinâmica das perturbações. Esta idéia foi o fundamento para anular termos decorrentes das perturbações, que estariam presentes no erro de observação. Uma realimentação de estados estimados foi considerada como ilustração de uma aplicação para o ODP proposto. Resultados numéricos foram apresentados para um exemplo de sistema descritor, onde foi utilizado o algoritmo de cálculo do ODP, elaborado com base nos resultados teóricos anteriores.

\section{AGRADECIMENTOS:}

Parte deste trabalho foi desenvolvido no LAAS-CNRS, Toulouse-França, durante doutorado sanduíche e pósdoutorado dos dois primeiros autores, respectivamente, com 


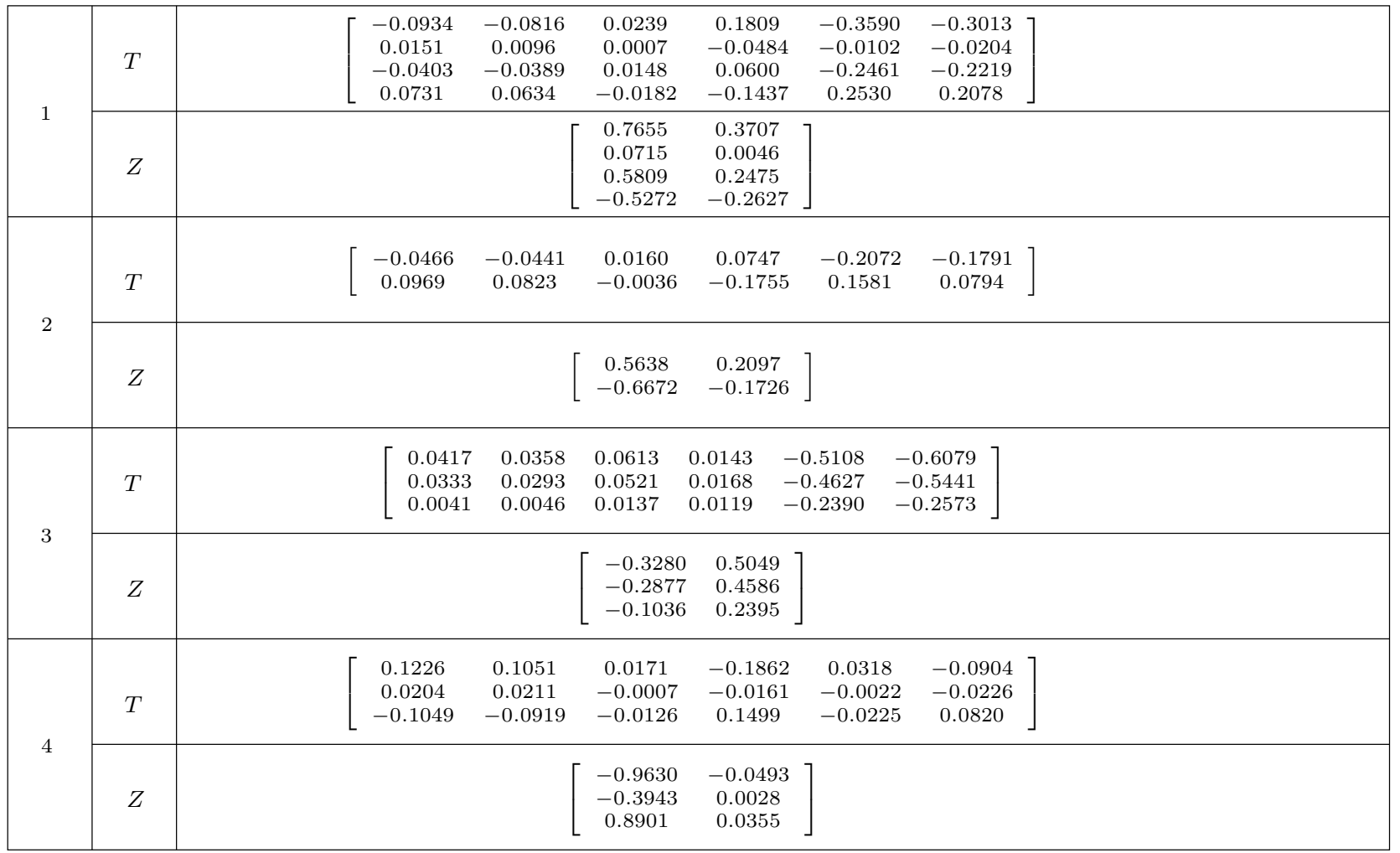

Tabela 2: Pares de matrizes $T$ e $Z$ do observador.

suporte financeiro da CAPES, Brasil. Este trabalho também contou com o suporte financeiro do CNPq, Brasil. Uma versão preliminar do presente artigo foi apresentada no CBA 2004, Gramado-R.S. Os autores agradecem ao editor associado e aos revisores por seus comentários, decorrentes do processo de revisão do artigo.

\section{APÊNDICE: EXPRESSÃO PARA O SISTEMA EM MALHA FECHADA SOB UMA REALIMENTAÇÃO DE ESTADOS ESTIMADOS}

Combinando (6) com (24), obtém-se

$$
u(t)=F S z(t)+F \bar{N} \bar{y}+F \tilde{N} \tilde{y}+v(t)
$$

Substituindo (18) e (19) em (37), obtém-se

$$
u(t)=F S z(t)+F \bar{N} \bar{Q} L A x(t)+F \tilde{N} \tilde{Q} C x(t)+v(t)
$$

A equação (22) pode ser reescrita como

$$
\tilde{N} \tilde{Q} C=I_{n}-\bar{N} \bar{Q} L A-S T E
$$

Substituindo (39) em (38), após breve simplificação, chegase a

$$
u(t)=(F-F S T E) x(t)+F S z(t)+v(t)
$$

Substituindo (40) em (1) e realizando alguns passos algébricos, encontra-se:

$$
E \dot{x}(t)=(A+B F) x(t)+B F S \varepsilon+B v+B_{w} w(t)
$$

A partir de (2), (15) e (41) é obtido o modelo do sistema em malha fechada dado por (25)-(26).

\section{REFERÊNCIAS}

Bhattacharyya, S. O. (1978). Observer design for linear systems with unknown inputs, IEEE Trans. on Automatic Control 23: 483-484.

Bolivar, A. R. (2001). Sur la synthèse de filtres de detection de defaillances, These doctorat de l'Universite Toulouse III, Spécialité automatique, LAAS du CNRS.

Castelan, E. B. (2005). Estabilização de sistemas descritores por realimentação de saídas via subespaços invariantes (aceito), Controle e Automação - SBA (5).

Castelan, E. B. and Silva, V. G. (2005). On the solution of a sylvester equation appearing in descriptor systems control theory, Systems \& Control Letters, accepted for publication 54: 109-117. 

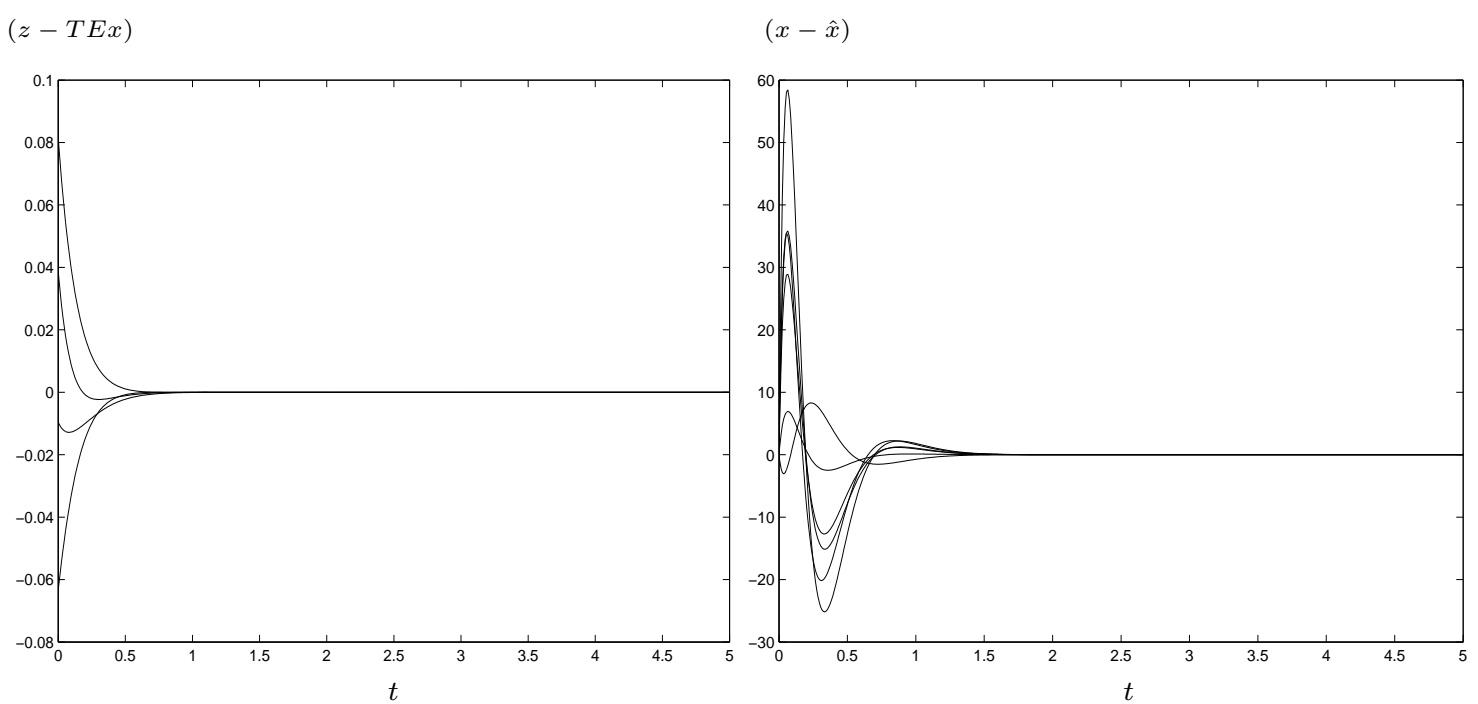

(a)
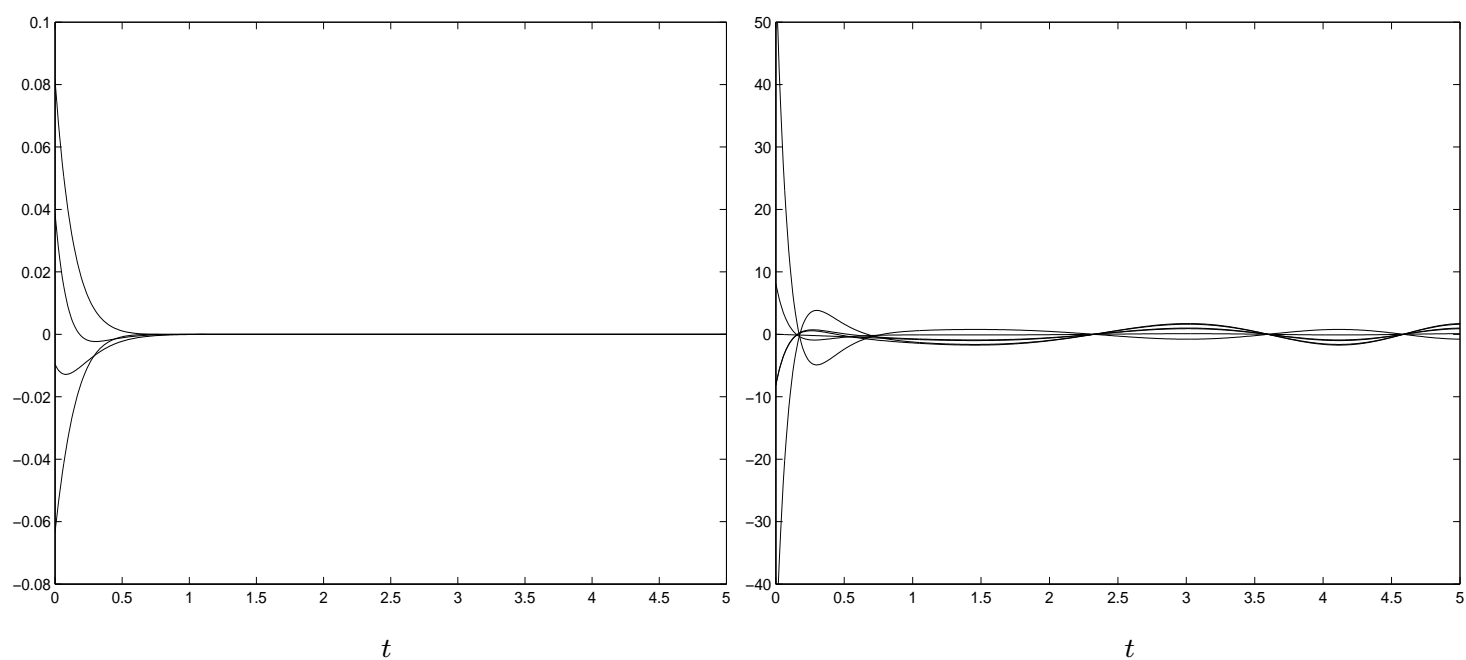

(b)

Figura 2: Curvas dos erros de estimação e observação para: (a) ODP e (b) observador com desacoplamento parcial.

Castelan, E. B., Silva, V. G., Tarbouriech, S. and Garcia, G. (2004). Disturbance decoupled minimal-order observers for a class of linear descriptor systems, Proc. of IFAC Symposium on Systems Structure and Control SSSC'04.

Chen, C. T. (1999). Linear System Theory and Design, Oxford University Press.

Chu, D. and Mehrmann, V. (1999a). Disturbance decoupled observer design for descriptor systems, Systems \& Control Letters 38: 37-48.

Chu, D. and Mehrmann, V. (1999b). Disturbance decoupled observer design for descriptor systems, Systems \& Control Letters 38: 37-48.

Dai, L. (1989). Singular Control Systems. Lecture Notes in Control and Information Sciences, Springer-Verlag.
Darouach, M. and Boutayeb, M. (1995). Design of observers for descriptor systems, IEEE Trans. on Automatic Control 40(7): 1323-1327.

Darouach, M., Zasadzinski, M. and Hayar, M. (1996). Reduced-order observer design for descriptor systems with unknown inputs, IEEE Trans. on Automatic Control 41(7): 1068-1072.

Hou, M. and Muller, P. C. (1992a). Design of observers for linear systems with unknown inputs, IEEE Trans. on Automatic Control 37: 871-875.

Hou, M. and Muller, P. C. (1992b). Observer for linear descriptor systems with unknown inputs control, Auto matisierungstechnic 40: 220-227. 
Johnson, C. D. (1975). On observers for linear systems with unknown and inaccessible inputs, International Journal of Control 21: 825-831.

Koenig, D. and Mammar, S. (2002). Design of proportionalintegral observer for unknown input descriptor systems, IEEE Trans. on Automatic Control 47(12): 2057-2062.

Liu, G. P. and Patton, R. J. (1998). Eigenstructure Assignment for Control System Design, John Wiley and Sons.

Muller, P. and Hou, M. (1993). On the observer design for descriptor systems, IEEE Trans. on Automatic Control 38(11): 1666-1671.

Nagpal, K. M. and Khargonekar, P. P. (1991). Filtering and smoothing in an $\mathcal{H}_{\infty}$ setting, IEEE Trans. on Automatic Control 36: 152-166.

Paraskevopoulos, P. N., Koumboulis, F. N., Tzierakis, K. G. and Panagiotakis, G. E. (1992). Observers design for generalized state space systems with unknown inputs, Systems \& Control Letters 18: 309-321.

Silva, V. G. (2005). Sobre síntese de observadores para sistemas descritores, Programa de pós graduação em engenharia elétrica, ppgeel, Universidade Federal de Santa Catarina-UFSC, Campus Universitário, Trindade, Florianópolis-SC. Tese de doutorado.

Syrmos, V. L. (1993). Computational observer design techniques for linear systems with unknown inputs using the concept of transmission zeros, IEEE Trans. on Automatic Control 38(5): 790-794.

Varga, A. (1995). On stabilization methods of descriptor systems, Systems \& Control Letters 24: 133-138.

Verhaegen, M. H. and dooren, P. V. (1986). A reduced observer for descriptor systems, Systems \& Control Letters 8(5): 29-37.

Yaesh, I. and Shaked, U. (1992). Game theory approach to optimal linear state estimation and its relation to $\mathcal{H}_{\infty}$ norm estimation, IEEE Trans. on Automatic Control 37: $828-831$.

Yang, C. W. and Tan, H. L. (1989). Observer design for singular systems with unknown inputs, International Journal of Control 49: 1937-1946. 